MRS. LEONA ISABELLA VON HESSEN (Orcid ID : 0000-0003-2851-5068)

DR. HELEN REEVES (Orcid ID : 0000-0003-0359-9795)

Article type : Original Articles

Corresponding Author Email ID: Jean-Francois.Dufour@insel.ch

\title{
HIGH SUBCUTANEOUS ADIPOSE TISSUE DENSITY CORRELATES NEGATIVELY WITH SURVIVAL IN PATIENTS WITH HEPATOCELLULAR CARCINOMA
}

\author{
von Hessen, Leona ${ }^{1,2}$, Roumet, Marie ${ }^{3}$, Maurer, Martin Helmut ${ }^{4}$, Lange, Naomi ${ }^{1}$, Reeves, Helen ${ }^{5,6}$, \\ Dufour, Jean-François ${ }^{1,2}$ and Radu, luliana-Pompilia ${ }^{1,2}$ \\ 1 Hepatology, Department of Clinical Research, University of Bern, Bern, Switzerland \\ 2 University Clinic for Visceral Surgery and Medicine, Inselspital Bern, Bern, Switzerland \\ ${ }^{3}$ Clinical Trials Unit Bern, Department of Clinical Research, University of Bern, Bern, Switzerland
}

${ }^{4}$ Department of Radiology, Inselspital, University of Bern, Bern, Switzerland

${ }^{5}$ The Northern Institute for Cancer Research, Newcastle University, Newcastle-upon-Tyne, UK

${ }^{6}$ The Liver Unit, Freeman Hospital, Newcastle-upon-Tyne Hospitals NHS Foundation Trust, Newcastle upon Tyne, UK

This article has been accepted for publication and undergone full peer review but has not been through the copyediting, typesetting, pagination and proofreading process, which may lead to differences between this version and the Version of Record. Please cite this article as doi: $\underline{10.1111 / L I V .14755}$

This article is protected by copyright. All rights reserved 
Word count main body of manuscript: 3937

Figures and tables: total of 3

\begin{abstract}
Abbreviations
HCC, hepatocellular carcinoma; OS, overall survival; HU, Hounsfield units; SM, skeletal muscle; SAT, subcutaneous adipose tissue; VAT, visceral adipose tissue; IMAT, intermuscular adipose tissue; CT, computed tomography; L3, third lumbar vertebra; HR, Hazard Ratio; BMI, body mass index; EASL, European Association for the Study of the Liver; BCLC, Barcelona Clinic Liver Cancer; HBV, hepatitis B virus; $\mathrm{HCV}$, hepatitis $\mathrm{C}$ virus; NAFLD, non-alcoholic fatty liver disease; OE, other etiology; NK, unknown etiology; MELD, Model of end-stage liver disease; ECOG, Eastern Cooperative Oncology Group; IQR, interquartile range; BAT, brown adipose tissue; VEGF, vascular endothelial growth factor
\end{abstract}

\title{
Conflict of interest
}

The authors declare that there is no conflict of interest regarding the publication of the manuscript.

\section{Funding Sources}

Swissliver - Stiftung für Leberkrankheiten

\section{Disclosure Statement}

Jean-François Dufour: Advisory committees: Abbvie, Bayer, BMS, Falk, Genfit, Genkyotex, Gilead Science, HepaRegenix, Intercept, Lilly, Merck, Novartis. Speaking and teaching: Abbvie, Bayer, BMS, Genfit, Gilead Science, Novartis

\section{Acknowledgement}

We would like to acknowledge Andrea Cavelti our dedicated HCC nurse for her constant support of the patients and their families.

This article is protected by copyright. All rights reserved 


\section{ABSTRACT}

\section{Background \& Aims}

Body composition parameters have been reported to add information, which can lead to tailored treatment and prognostication for oncological patients. Data for patients with hepatocellular carcinoma (HCC) are scarce. We assessed the association between different body composition parameters and overall survival (OS) in two different newly diagnosed HCC populations.

\section{Methods}

The area $\left(\mathrm{cm}^{2}\right)$ and density (Hounsfield Units $(\mathrm{HU})$ ) of skeletal muscle (SM) and adipose tissue (subcutaneous (SAT), visceral (VAT) and intermuscular (IMAT)) were measured on computed tomography (CT) scans at the level of the third lumbar vertebra (L3) in two cohorts of patients diagnosed in different HCC stages (Bern, Switzerland $n=187$ and Newcastle, United Kingdom $\mathrm{n}=216$ ). Univariate and multivariate Cox regressions analyses were used to assess the crude and adjusted association of body composition parameters with OS.

\section{Results}

By univariate analysis, in both cohorts, Bern and Newcastle, high SAT density (Hazard Ratio $(H R): 1.35 ; 1.12-1.62, p<0.001$ and $1.44 ; 1.27-1.63, p<0.001$, respectively) and high VAT density (HR: 1.38; 1.1-1.72, $p=0.005$ and HR: 1.53; 1.3-1.81, $p<0.001$, respectively) correlated negatively with survival. After model adjustment for potential baseline confounders (gender, age, diabetes, cirrhosis, MELD score, BCLC stage) in a multivariate analysis, SAT density remained associated with mortality in Bern and Newcastle (Bern: HR: 1.27; 1.04-1.57, p=0.022; Newcastle: HR: $1.23 ; 1.03-1.48, p=0.022)$ and VAT remained associated with mortality in Bern (HR: 1.31; 1.05-1.65, $p=0.019)$.

\section{Conclusions}

Based on two HCC cohorts, our data show that high SAT density correlates negatively with OS in HCC patients.

\section{Word count: 250}

Key words: body composition, analytic morphomics, computed tomography, outcome 


\section{LAY SUMMARY}

Altered body composition, including loss of muscle mass and distribution of fat tissue, has been reported to negatively impact survival of patients with various cancers, while data for patients with liver cancer are scarce.

The results of our study show that high subcutaneous fat tissue density, which can bring important information about the quality of the tissue, measured by CT scan, is associated with shorter survival in patients with newly diagnosed liver cancer. It can easily be determined in clinical practice, as CT examinations are routinely performed in patients with liver cancer.

This article is protected by copyright. All rights reserved 


\section{INTRODUCTION}

In recent years, several studies revealed that altered body composition is associated with survival in several malignancies ${ }^{1}$. Compared to commonly used anthropometry, such as body mass index (BMI) or waist circumference, measurements on CT scans allow an adequate differentiation of body composition components, including visceral and subcutaneous fat distribution, and are considered as the gold standard technique for assessing skeletal muscle mass ${ }^{2-4}$. Analytic morphomics is an attractive and well-studied tool to evaluate the individual body composition in oncological patients and can easily be adopted in clinical practice, as imaging examinations are routinely performed during diagnosis, staging and follow up ${ }^{1}$.

The loss of muscle mass and strength, referred to as sarcopenia, is the most studied prognostic parameter in oncological patients. It has been associated with a reduced OS rate and higher recurrence rate in early-stage HCC patients, while studies addressing the prognostic role of body composition in advanced-stage HCC have shown conflicting results ${ }^{5-8}$. Most of the studies supporting a negative impact of low muscle mass on the prognosis of HCC patients were performed in Asian cohorts, while data for European patients are scarce.

Obesity as well as underweight have been associated with several malignancies including HCC ${ }^{9}$. In particular, the accumulation of VAT, referred to as visceral adiposity, has been identified as a significant risk factor for developing HCC and recurrence of HCC after liver transplantation ${ }^{10}$. However, its further impact on the course of the disease is not well studied, yet.

In addition to quantitative measurements of both skeletal muscle and adipose tissue areas, the analyses of the tissue density expressed in $\mathrm{HU}$ can bring important information about the quality of the tissue and indirectly offer an insight into the pathophysiology. Even though HU are easily determined on CT images, tissue density has received much less interest and little is known regarding its prognostic significance in HCC patients ${ }^{11}$.

In this study, we report the association of body composition features and OS in two different HCC populations.

\section{METHODS}

\section{Study populations}

This article is protected by copyright. All rights reserved 
In the current study, two different cohorts with newly diagnosed HCC patients were analysed.

The Bern cohort comprised all patients with HCC consecutively included in the prospective HCC cohort from the University Hospital Bern (Bern HCC Cohort), Switzerland. The local ethics committee (Kantonale Ethikkommission Bern, Bern, Switzerland) approved collection of patient information and the study protocol (project-ID 2017-00957), which was consistent with the principles of the current version of the Declaration of Helsinki. Consents from all of the patients were established prior to submission. All patients included in between November 2010 and October 2016 were initially reviewed $(n=294)$. Patients older than 18 years with CT scan available at time of diagnosis and European origin were selected $(n=187)$. Patients with MRI or without or incomplete CT scan available at time of diagnosis $(n=93)$ and non-European origin $(n=14)$ were excluded. Patients who underwent liver transplantation were censored at the time of transplantation $(n=25)$.

The second cohort comprised patients with HCC cared in the Newcastle upon Tyne Hospitals NHS Foundation Trust (Newcastle HCC cohort), United Kingdom. These included 632 patients that formed part of a prospective audit performed between June 2004 and December $2010{ }^{12}$, whose CT scan data were retrospectively collected if available, as approved by the London Bromley Research Ethics Committee (reference 18/LO/1429) and the Newcastle NHS Foundation Trust Research and Development Department (reference 8765). After exclusion criteria were similarly applied, a total of 216 patients were analyzed in the Newcastle cohort.

In all cases, HCC diagnosis was established on typical findings on contrast-enhanced multiphase imaging (hyperenhancement in the arterial phase and washout in the portal venous or delayed phases) or histopathology according to the diagnostic criteria of the European Association for the Study of the Liver (EASL) clinical practice guidelines ${ }^{13}$. The tumor stages were classified according to the Barcelona Clinic Liver Cancer (BCLC) staging system ${ }^{14}$. Cases in both cohorts were discussed in the multidisciplinary liver tumor boards at each institution.

\section{Clinical data collection}

Clinical data were collected from prospective databases and medical records of both cohorts. The following data were documented: patient demographics, anthropometrics (height, weight), clinical history, comorbidities, etiology of underlying liver disease (chronic hepatitis B virus (HBV) infection, chronic hepatitis $\mathrm{C}$ virus (HCV) infection, alcohol abuse, non-alcoholic fatty liver disease (NAFLD), other etiologies (OE) (haemochromatosis, primary biliary cholangitis, primary sclerosing cholangitis) and unknown etiology (NK)), presence of cirrhosis, Child-Pugh score, Model of end- 
stage liver disease (MELD) score, BCLC classification, Eastern Cooperative Oncology Group (ECOG) score, tumor characteristics, treatment modality, laboratory results and survival time. Liver function was staged according to the Child-Pugh criteria, including serum albumin and bilirubin levels, prothrombin time, presence of ascites and degree of hepatic encephalopathy. The MELD score calculation included serum bilirubin, creatinine and INR (international normalized ratio) values. The performance status was assessed by the ECOG score, which is widely used to quantify limitations in daily living activities of cancer patients ${ }^{15}$. Tumor staging was based on to the BCLC classification criteria, including tumor burden, liver function and performance status (early stage (BCLC 0 or $A$ ), intermediate stage (BCLC B), advanced stage (BCLC C) and terminal stage $(B C L C D))^{14}$.

\section{CT scan analysis of body composition components}

The CT scans had been performed for the purpose of HCC diagnosis (protocol with a previous non-contrast scan followed by contrast-enhanced arterial, portal venous and late venous contrast phases). A single unenhanced axial slice of each patient's CT scan was selected at the level of L3 to allow a standardized analysis of the same area in each patient. Areas $\left(\mathrm{cm}^{2}\right)$ of SM, IMAT, SAT and VAT were calculated using SliceOmatic ${ }^{\text {TM }}$ software (Tomovision $\odot$, Montreal, Canada, version 5.0). The imaging software enables semi-automated tissue segmentation and calculation based on HU thresholds. The following predefined HU ranges were used: -29 to $+150 \mathrm{HU}$ for SM, -150 to -50 for VAT and -190 to -30 for SAT and IMAT. Cross-sectional areas of each variable were then normalized for the patient's height $\left(\mathrm{m}^{2}\right)$ to obtain indexes $\left(\mathrm{cm}^{2} / \mathrm{m}^{2}\right)$. In addition, the density of each tissue was documented in HU. The inter-observer reproducibility was assessed by analysing CT scans of 40 patients randomly selected from the cohorts by two trained observers, L.v.H. and N.L. The inter-observer reproducibility was $97.3 \%$. The skeletal muscle area at the level of L3 includes the psoas, rectus abdominus, internal and external obliques, transversus abdominis, quadratus lumborum and erector spinae muscles. Subcutaneous, visceral and intermuscular adipose tissues were analyzed separately.

\section{Statistical analyses}

Patient's characteristics from Bern and Newcastle were compared by the t-test for continuous data or by the non-parametric Wilcoxon rank sum test when normality assumption was not satisfied. For categorical data, Fisher's exact test was used if expected frequencies were lower than five in any cell, else the chi-squared test was applied. Within each cohort, we also reported and compared the body composition features of patients at different BCLC stages and of patients belonging to the different Child-Pugh classes. Differences between BCLC stages and Child-Pugh 
classes were tested using one-way ANOVA or Kruskal-Wallis test when test assumptions were not met. Post-hoc analysis (Tukey test after an ANOVA or Dunn test after the Kruskal-Wallis test) was performed when a significant effect was found in the two cohorts.

In our model we kept the body parameters as continues data, in order to avoid the loss of information (aggregation risk). Moreover, even though in the literature there are published thresholds, there is no agreement $16,17,18$. The effect of each of the body composition variables (SM, VAT, SAT, IMAT) on the OS was first analyzed by categorizing the data into two categories. Survival in the low (i.e. below the median) and high (above the median) category was analyzed using Kaplan-Meier curves and compared using a log-rank test. We then considered each of the four-studied body composition variables as a continuous variable and evaluated their effect on survival by using Cox proportional hazards model. We first assessed the crude (unadjusted) association between body composition and survival and then adjusted the model for potential baseline confounders by including sex, age, diabetes, presence of cirrhosis, MELD score, and BCLC stage.

All analyses were performed using R 3.3.1 software.

\section{RESULTS}

\section{Patient characteristics}

The Bern cohort comprised 187 patients with a male majority population (83.4\%), with a median age of 64 (interquartile range (IQR) 57-70.5) and a median BMI of 26.6 (IQR 23.9-29.9). Alcohol abuse $(31 \%)$ was the main etiology followed by chronic HCV infection (27.4\%) and other etiologies (Table 1$)$. The majority of patients had cirrhosis $(82.4 \%)$, Child-Pugh class A liver function (60.5\%) and BCLC stage A HCC (38\%). The curative intention treatment (i.e transplant, resection or ablative therapy) was the main approach with $42.8 \%$, followed by non-curative therapy (i.e. chemoembolization and sorafenib) in $30.5 \%$. Only $10.2 \%$ were assessed to best supportive care.

The Newcastle cohort comprised 216 patients with a male majority population (82.4\%), with a median age of 70 (IQR 64-76) and a median BMI of 28.3 (IQR 25.1-33.1). The most common etiology was NAFLD (31\%) followed by alcohol abuse (25.5\%) and other etiologies (Table 1). In this cohort, the majority of patients had cirrhosis (70\%), Child-Pugh class A liver function $(57.7 \%)$ 
and BCLC stage C HCC (38.4\%). Of 151 patients with available treatment data, $41.7 \%$ underwent non-curative therapy and $29.1 \%$ benefited of curative therapy, respectively.

The body composition features are summarized in Table 1. Median SAT index and density, IMAT index and muscle index were similar in the two populations. The Newcastle cohort patients had a lower muscle density (32.6 vs. $36.3, p<0.001)$ in keeping with more fatty infiltration of muscle. In addition, the Newcastle patients had a higher VAT index (69.4 vs. 54, $p=0.025)$ and a higher VAT density $(-90$ vs. $-87, p=0.003)$, suggesting a predominance of visceral obesity in the Newcastle cohort.

\section{Differences in body composition parameters among BCLC stages and Child-Pugh classes} In the Bern cohort IMAT density was not found to be affected either by BCLC stages or by ChildPugh classes. A significant effect of BCLC stages on IMAT index was detected in the Newcastle cohort $(p=0.027)$, where IMAT index values varied non-linearly (i.e. values tended to increase between stages $A$ and $B$, decrease between stage $B$ and $C$ and increase between stage $C$ and D).

No association of either BCLC or Child-Pugh stages with muscle mass index was detected in the Bern cohort. However, in the Newcastle cohort, muscle density values decreased across BCLC stages $(p=0.005)$.

In both cohorts, Bern and Newcastle, we found a significant variation of VAT density across Child-Pugh classes and of SAT density among BCLC stages. Both body parameters tended to increase across Child-Pugh classes and BCLC stages, respectively (sup. Tables 1 and 2; sup. Fig. 1 and 2).

\section{Association between baseline body composition parameters and survival}

At the time of analysis, in both cohorts, Bern and Newcastle, after a median follow-up of 649 (IQR $307-1210)$ and 324 days (IQR 107-874), respectively, 112 (59.9\%) and 176 (81.5\%) patients had died, respectively.

We used two approaches to investigate the effect of VAT and SAT on survival. First, we performed a descriptive analysis and classified the patients within the cohorts according to the body composition parameters - median values - into two groups: below and equal or above the 
median. In both cohorts, patients from the low SAT density category had a better survival curve than patients from the higher category $(p=0.042$ and $p=0.003$, log-rank test, in Bern and Newcastle, respectively) (Fig.1).

However, we did not detect any significant difference in survival of patients of the low and high muscle density categories as well as of the low and high IMAT density categories.

\section{The effect of each body composition parameter on survival of patients with HCC}

In a second step, in order to quantify the effect of body composition on survival, we kept the body composition as continuous and performed crude and adjusted Cox proportional hazards regression analysis (Table 2 ).

Results of the crude analysis for VAT and SAT density confirmed the occurrence of a significant negative effect of these variables on survival. In Bern cohort both SAT density and VAT density were independently associated with mortality $(\mathrm{HR} 1.35,95 \% \mathrm{Cl} 1.12,1.62: p=0.001)$ and $(\mathrm{HR}$, 1.44 95\%Cl: 1.27, 1.63, p<0.001), respectively. Similar in Newcastle cohort (HR 1.38, 95\%Cl: $1.38,1.1,1.72, p=0.005)$ and $(\operatorname{HR} 1.53,95 \% \mathrm{Cl} 1.3,1.81, \mathrm{p}<0.001)$.

When adjusting our model for patient characteristics (age, sex), comorbidities (diabetes), tumor stage (BCLC), presence of cirrhosis and hepatic function (MELD), the effect of SAT density lowered down but remained significant in Bern (HR 1.27,95\% 1.04, 1.57, $p=0.022)$ as well as in Newcastle (HR 1.23, 95\% 1.03, 1.48, $p=0.022$ ). However, the effect of VAT remained significant only in Bern (HR 1.31, 95\% 1.05, 1.65, $p=0.019)$.

In the Newcastle cohort we also detected a significant crude association between survival and muscle index $(\mathrm{HR} 0.79,95 \% \mathrm{Cl} 0.62,0.99, \mathrm{p}=0.045)$, which did not remain significant when adjusting the model ( $\mathrm{HR} 0.83,95 \% \mathrm{Cl} 0.51,1.33, \mathrm{p}=0.43)$.

No significant association between survival and SAT index, VAT index, IMAT (index/density) and muscle (index/density) was detected in the studied cohorts.

\section{DISCUSSION}

We have evaluated the correlation between body composition at $L 3$ and survival of two cohorts of patients (Bern and Newcastle HCC cohorts) that had been newly diagnosed in various HCC 
stages. Our study showed that higher abdominal SAT density, assessed on CT, was associated with mortality in patients with HCC, with adjustment for risk factors including age, tumor stage (BCLC), presence of cirrhosis, hepatic function (MELD) and comorbidities (diabetes).

There is significant evidence suggesting a correlation between obesity and several types of cancer. Although, BMI is one of the most widely used tools for the classification of obesity, it is an imprecise tool to assess the body composition, i.e. the amount and distribution of muscle and specific adipose tissue compartments and other components of weight such as water, bone and organs ${ }^{19}$. Analytic morphomics allows a more accurate evaluation of body components in terms of volume and density (quality) that might help us better understand the underlying patient features (phenotype) ${ }^{20}$. This is particularly important in HCC, in which not only the tumor but also the underlying liver disease has an impact on the prognosis of these patients. Moreover, in cirrhotic and HCC patients, CT scan is often used for follow-up; therefore, it could be used simultaneously to provide a precise, objective, and reproducible quantification of body composition parameters.

In recent years, sarcopenia was one of the most evaluated body composition components and its presence has proven to be correlated with poor prognosis, recurrence and overall complications in patients with $\mathrm{HCC}$ in the early phase ${ }^{7,21}$. In contrast with previous studies, in our study, the muscle index and density had no impact on survival. The reason for this inconsistency is undoubtedly multi-factorial since sarcopenia was correlated with several characteristics, such as age, gender, liver function, BMI and physical activity ${ }^{7}$. Furthermore, it is not clear whether sarcopenia is a causal factor of poor prognosis, a concomitant finding of HCC or a type of comorbidity. In a study conducted by Sachdeva M., the authors showed that patients with cachexia tend to lose their weight more, when compared to patients with sarcopenia; therefore, the increase in mortality may be a consequence of cancer-related cachexia rather than sarcopenia ${ }^{22}$. The low muscle density has been shown to be associated with insulin resistance in obese nondiabetic patients and patients with diabetes type II; this might explain why in the Newcastle cohort the muscle density was lower compared to the Bern cohort ${ }^{23}$. Although sarcopenia was considered the hallmark of cancer cachexia, recent studies showed that adipose tissue is the main player in this process ${ }^{24}$. Nonetheless, the adipose tissue (density and quantity) has received much less attention, so the results are scarce and conflicting.

Two previous large community-based cohort studies reported a negative correlation between SAT and VAT density and survival, which demonstrated that an increased adipose tissue 
attenuation (VAT and SAT) might serve as a biomarker for all-cause mortality, including cancer mortality ${ }^{25,26}$. For extremity sarcomas, it was shown that an increase in SAT density was associated with increased overall mortality ${ }^{27}$. On the other hand, Delouya et al. found that lower SAT density showed a trend toward more aggressive cancers, whereas VAT was a protective factor ${ }^{28}$.

In both HCC cohorts, we observed that high SAT density had a negative impact on survival. Several hypothesizes might explain this finding.

Although CT does not directly provide any functional data, the radio-density of adipose tissue, which is determined by the ratio of fat to water, can distinguish different types of adipose tissues 29. Indeed, Baba and colleagues have demonstrated a concordant increase in CT attenuation (toward greater radio-density), with decreased lipid content and the activation of the brown adipose tissue (BAT), suggesting that in some adipose tissue subtypes, changes in attenuation may accompany alterations in lipid concentration ${ }^{30}$. Moreover BAT is rich in vascularisation, which lead to an increase in radio-density, due to the tissue properties of blood ${ }^{31}$. The vascular endothelial growth factor (VEGF) is not only a key factor in angiogenesis, but also has pleiotropic effects on several nonvascular cells. Previous studies have shown that an over-expression of VEGF leads to a browning of white adipocytes, and to an increased BAT mass ${ }^{32}$. In addition to playing a crucial role in HCC development and metastasis, VEGF is also responsible for diabetic retinopathy development ${ }^{33}$. In a study conducted by Azuma, the authors have noted that diabetic retinopathy is a risk factor associated with the development of HCC in NAFLD patients ${ }^{34}$. In the light of these findings, we speculate that overexpression of VEGF in HCC patients might be one of the factors implied in the browning process of SAT. Furthermore, previous studies have shown that SAT is more sensitive to the browning process than VAT in both mice and humans ${ }^{35}$. Another explanation for a higher adipose tissue density is the pro-inflammatory state that was observed in oncological patients. In a recent study the authors reported that SAT tissue of patients with gastro-intestinal cancer is characterized by fibrosis and inflammatory cell infiltration 36. On CT studies these changes correspond to an increased SAT density ${ }^{25}$.

The VAT density variability across Child-Pugh classes, namely between class $A$ and $B$ is in agreement with the result of Parikh and colleagues, who showed that VAT could be used as a surrogate for the prediction of early portal hypertension ${ }^{37}$. The authors suggested that the changing of VAT density might be the result of either increasing the portal shunts or the interstitial 
edema. Taken together, these findings suggest that fat density may be an important radio-marker for HCC patients but warrants further investigation.

The major limitation of the present study was its retrospective analysis of prospectively acquired data. This characteristic limited the assessment of various clinical and paraclinical data (i.e. BMI, Child Pugh score, BCLC stage, comorbidities, treatment), especially in the Newcastle cohort, in which only 216 of 632 were analyzed due to the lack of CT data availability. Besides, some of these patients were included in the pre-Sorafenib era.

Even though the Newcastle cohort is not representative for the general HCC population, it is representative for an HCC population, which is becoming more common (i.e. older patient with comorbidities), hence our intention to analyse it. Because we observed associations, we cannot claim any prognostic effect of SAT density in our population. Future longitudinal studies are required to clarify this issue further. The differences between the two populations in terms of baseline characteristics did not allow us to validate the results achieved. Nevertheless, this feature of our cohorts, differences in terms of baseline characteristics, strengthens our findings.

\section{CONCLUSION}

Based on two HCC cohorts, our data show that high SAT density correlates negatively with OS in HCC patients. 


\section{REFERENCES}

1. Yip C, Dinkel C, Mahajan A, Siddique M, Cook GJR, Goh V. Imaging body composition in cancer patients: visceral obesity, sarcopenia and sarcopenic obesity may impact on clinical outcome. Insights Imaging. 2015;6:489-497.

2. Borga M, West J, Bell JD, et al. Advanced body composition assessment: from body mass index to body composition profiling. J Investig Med Off Publ Am Fed Clin Res. 2018;66:1-9.

3. Shuster A, Patlas M, Pinthus JH, Mourtzakis M. The clinical importance of visceral adiposity: a critical review of methods for visceral adipose tissue analysis. Br J Radiol. 2012;85:1-10.

4. Cruz-Jentoft AJ, Baeyens JP, Bauer JM, et al. Sarcopenia: European consensus on definition and diagnosis: Report of the European Working Group on Sarcopenia in Older People. Age Ageing. 2010;39:412-423.

5. Labeur TA, van Vugt JLA, Ten Cate DWG, et al. Body Composition Is an Independent Predictor of Outcome in Patients with Hepatocellular Carcinoma Treated with Sorafenib. Liver Cancer. 2019;8:255-270.

6. Levolger S, van Vledder MG, Muslem R, et al. Sarcopenia impairs survival in patients with potentially curable hepatocellular carcinoma. J Surg Oncol. 2015;112:208-213.

7. Zhang G, Meng S, Li R, Ye J, Zhao L. Clinical significance of sarcopenia in the treatment of patients with primary hepatic malignancies, a systematic review and meta-analysis. Oncotarget. 2017;8:102474-102485.

8. Saeki I, Yamasaki T, Maeda M, et al. No Muscle Depletion with High Visceral Fat as a Novel Beneficial Biomarker of Sorafenib for Hepatocellular Carcinoma. Liver Cancer. 2018;7:359371.

9. Tateishi R, Okanoue T, Fujiwara N, et al. Clinical characteristics, treatment, and prognosis of non-B, non-C hepatocellular carcinoma: a large retrospective multicenter cohort study. J Gastroenterol. 2015;50:350-360.

10. Montano-Loza AJ, Mazurak VC, Ebadi M, et al. Visceral adiposity increases risk for hepatocellular carcinoma in male patients with cirrhosis and recurrence after liver transplant. Hepatol Baltim Md. 2018;67:914-923.

11. Charette N, Vandeputte $C$, Ameye $L$, et al. Prognostic value of adipose tissue and muscle mass in advanced colorectal cancer: a post hoc analysis of two non-randomized phase II trials. BMC Cancer. 2019;19:134.

12. Dyson JK, Anstee QM, McPherson S. Non-alcoholic fatty liver disease: a practical approach to diagnosis and staging. Frontline Gastroenterol. 2014;5:211-218. 
13. European Association for the Study of the Liver. Electronic address: easloffice@easloffice.eu, European Association for the Study of the Liver. EASL Clinical Practice Guidelines: Management of hepatocellular carcinoma. J Hepatol. 2018;69:182-236.

14. Llovet JM, Brú C, Bruix J. Prognosis of hepatocellular carcinoma: the BCLC staging classification. Semin Liver Dis. 1999;19:329-338.

15. Nishikawa H, Kita R, Kimura T, et al. Clinical Implication of Performance Status in Patients with Hepatocellular Carcinoma Complicating with Cirrhosis. J Cancer. 2015;6:394-402.

16. Antonelli G, Gigante E, lavarone M, et al. Sarcopenia is associated with reduced survival in patients with advanced hepatocellular carcinoma undergoing sorafenib treatment. United European Gastroenterol J. 2018;6:1039-1048.

17. Imai K, Takai K, Watanabe S, et al. Sarcopenia Impairs Prognosis of Patients with Hepatocellular Carcinoma: The Role of Liver Functional Reserve and Tumor-Related Factors in Loss of Skeletal Muscle Volume. Nutrients. 2017;9:1054.

18. European Association for the Study of the Liver. Electronic address: easloffice@easloffice.eu; European Association for the Study of the Liver. EASL Clinical Practice Guidelines on nutrition in chronic liver disease. J Hepatol. 2019;70(1):172-193.

19. Strulov Shachar S, Williams GR. The Obesity Paradox in Cancer-Moving Beyond BMI. Cancer Epidemiol Biomark Prev Publ Am Assoc Cancer Res Cosponsored Am Soc Prev Oncol. 2017;26:13-16.

20. Singal AG, Zhang P, Waljee AK, et al. Body Composition Features Predict Overall Survival in Patients With Hepatocellular Carcinoma. Clin Transl Gastroenterol. 2016;7:e172.

21. Chang K-V, Chen J-D, Wu W-T, Huang K-C, Lin H-Y, Han D-S. Is sarcopenia associated with hepatic encephalopathy in liver cirrhosis? A systematic review and meta-analysis. J Formos Med Assoc Taiwan Yi Zhi. 2019;118:833-842.

22. Sachdeva M, Chawla YK, Arora SK. Immunology of hepatocellular carcinoma. World J Hepatol. 2015;7:2080-2090.

23. Kalafateli M, Karatzas A, Tsiaoussis G, et al. Muscle fat infiltration assessed by total psoas density on computed tomography predicts mortality in cirrhosis. Ann Gastroenterol. 2018;31:491-498.

24. Daas SI, Rizeq BR, Nasrallah GK. Adipose tissue dysfunction in cancer cachexia. J Cell Physiol. 2018;234:13-22.

25. Murphy RA, Register TC, Shively CA, et al. Adipose tissue density, a novel biomarker predicting mortality risk in older adults. J Gerontol A Biol Sci Med Sci. 2014;69:109-117.

26. Rosenquist KJ, Massaro JM, Pedley A, et al. Fat quality and incident cardiovascular disease, all-cause mortality, and cancer mortality. J Clin Endocrinol Metab. 2015;100:227-234.

This article is protected by copyright. All rights reserved 
27. Veld J, Vossen JA, De Amorim Bernstein K, Halpern EF, Torriani M, Bredella MA. Adipose tissue and muscle attenuation as novel biomarkers predicting mortality in patients with extremity sarcomas. Eur Radiol. 2016;26:4649-4655.

28. Delouya G, Tiberi D, Bhatnagar SR, Campeau S, Saad F, Taussky D. Impact of adipose tissue on prostate cancer aggressiveness - analysis of a high-risk population. Horm Mol Biol Clin Investig. 2018;36.

29. Frankl J, Sherwood A, Clegg DJ, Scherer PE, Öz OK. Imaging Metabolically Active Fat: A Literature Review and Mechanistic Insights. Int J Mol Sci. 2019;20.

30. Baba S, Jacene HA, Engles JM, Honda H, Wahl RL. CT Hounsfield units of brown adipose tissue increase with activation: preclinical and clinical studies. J Nucl Med Off Publ Soc Nucl Med. 2010;51:246-250.

31. Furlan A, Fakhran S, Federle MP. Spontaneous abdominal hemorrhage: causes, CT findings, and clinical implications. AJR Am J Roentgenol. 2009;193:1077-1087.

32. Bagchi M, Kim LA, Boucher J, Walshe TE, Kahn CR, D'Amore PA. Vascular endothelial growth factor is important for brown adipose tissue development and maintenance. FASEB J Off Publ Fed Am Soc Exp Biol. 2013;27:3257-3271.

33. Simó R, Sundstrom JM, Antonetti DA. Ocular Anti-VEGF therapy for diabetic retinopathy: the role of VEGF in the pathogenesis of diabetic retinopathy. Diabetes Care. 2014;37:893-899.

34. Azuma S, Asahina Y, Kakinuma S, et al. Diabetic Retinopathy as a Risk Factor Associated with the Development of Hepatocellular Carcinoma in Nonalcoholic Fatty Liver Disease. Dig Dis. 2019;37:247-254.

35. Rosell M, Kaforou M, Frontini A, et al. Brown and white adipose tissues: intrinsic differences in gene expression and response to cold exposure in mice. Am J Physiol Endocrinol Metab. 2014;306:E945-964.

36. Batista ML, Henriques FS, Neves RX, et al. Cachexia-associated adipose tissue morphological rearrangement in gastrointestinal cancer patients. J Cachexia Sarcopenia Muscle. 2016;7:37-47.

37. Parikh ND, Zhang P, Singal AG, et al. Body Composition Predicts Survival in Patients with Hepatocellular Carcinoma Treated with Transarterial Chemoembolization. Cancer Res Treat Off J Korean Cancer Assoc. 2018;50:530-537.

Table 1. Demographic and clinical characteristics of Bern cohort and Newcastle cohort 


\begin{tabular}{|c|c|c|c|c|c|c|c|}
\hline \multirow[t]{2}{*}{ Characteristics } & \multicolumn{2}{|r|}{$\begin{array}{l}\text { Overall } \\
(n=403)\end{array}$} & \multicolumn{2}{|r|}{$\begin{array}{l}\text { Bern cohort } \\
\qquad(n=187)\end{array}$} & \multicolumn{2}{|c|}{$\begin{array}{l}\text { Newcastle cohort } \\
\qquad(n=216)\end{array}$} & \multirow[t]{2}{*}{ P-value } \\
\hline & $\mathbf{N}$ & & $\mathbf{N}$ & & $\mathbf{N}$ & & \\
\hline Male & 403 & $334(82.9 \%)$ & 187 & 156 (83.4\%) & 216 & $178(82.4 \%)$ & 0.446 \\
\hline Age, years & 403 & $67.0[60.0 ; 74.0]$ & 187 & $64.0[57.0 ; 70.5]$ & 216 & $70.0[64.0 ; 76.0]$ & $<0.001$ \\
\hline BMI & 325 & $27.2[24.2 ; 30.9]$ & 186 & $26.6[23.9 ; 29.9]$ & 139 & $28.3[25.1 ; 33.1]$ & $<0.001$ \\
\hline Etiology & 403 & & 187 & & 216 & & \\
\hline Alcohol & & $113(28 \%)$ & & $58(31 \%)$ & & $55(25.5 \%)$ & $<0.130$ \\
\hline $\mathrm{HCV}$ & & $69(17.1 \%)$ & & $51(27.3 \%)$ & & $18(8.3 \%)$ & $<0.001$ \\
\hline NAFLD & & $90(22.3 \%)$ & & $23(12.3 \%)$ & & $67(31 \%)$ & $<0.001$ \\
\hline HBV & & $29(7.2 \%)$ & & $25(13.4 \%)$ & & $4(1.9 \%)$ & $<0.001$ \\
\hline OE & & $32(7.9 \%)$ & & $11(5.9 \%)$ & & $21(9.7 \%)$ & $<0.107$ \\
\hline NK & & $70(17.4 \%)$ & & $19(10.2 \%)$ & & $51(23.6 \%)$ & $<0.001$ \\
\hline $\begin{array}{c}\text { Type of } \\
\text { comorbidity }\end{array}$ & 403 & $156(38.7 \%)$ & 187 & $55(29.4 \%)$ & 216 & $101(46.8 \%)$ & $<0.001$ \\
\hline & & & mor c & haracteristics & & & \\
\hline $\mathrm{BCLC}$ & 377 & & 187 & & 190 & & $<0.001$ \\
\hline 0 & & $7(1.9 \%)$ & & $7(3.7 \%)$ & & $0(0.0 \%)$ & \\
\hline A & & $107(28.4 \%)$ & & $71(38.0 \%)$ & & $36(16.7 \%)$ & \\
\hline$B$ & & $90(23.9 \%)$ & & $58(31.0 \%)$ & & $32(14.8 \%)$ & \\
\hline $\mathrm{C}$ & & $118(31.3 \%)$ & & $35(18.7 \%)$ & & $83(38.4 \%)$ & \\
\hline $\mathrm{D}$ & & $55(14.5 \%)$ & & $16(8.6 \%)$ & & $39(18.1 \%)$ & \\
\hline
\end{tabular}




\begin{tabular}{|c|c|c|c|c|c|c|c|}
\hline \multicolumn{8}{|c|}{ Liver function } \\
\hline Cirrhosis & 304 & $304(75.4 \%)$ & 187 & $154(82.4 \%)$ & 214 & $150(70 \%)$ & 0.006 \\
\hline $\begin{array}{c}\text { Child-Pugh } \\
\text { Class }\end{array}$ & 289 & & 152 & & 137 & & 0.016 \\
\hline$A$ & & $171(59.2 \%)$ & & $92(60.5 \%)$ & & $79(57.7 \%)$ & \\
\hline$B$ & & $87(30.1 \%)$ & & $51(33.6 \%)$ & & $36(26.3)$ & \\
\hline C & & $31(10.7 \%)$ & & $9(5.9 \%)$ & & $22(16.1 \%)$ & \\
\hline MELD-Na score & 295 & $9.00[7.00 ; 11.93]$ & 186 & $9.00[7.00 ; 11.00]$ & 109 & $10.00[7.70 ; 12.85]$ & 0.001 \\
\hline \multicolumn{8}{|c|}{ Body composition } \\
\hline Muscle index & 286 & $47.1[42.0 ; 52.5]$ & 187 & $47.2[42.2 ; 52.6]$ & 99 & $46.6[41.3 ; 52.4]$ & 0.84 \\
\hline IMAT index & 270 & $2.85[1.46 ; 4.77]$ & 171 & $2.78[1.43 ; 4.84]$ & 99 & $2.92[1.61 ; 4.33]$ & 0.95 \\
\hline VAT index & 269 & $58.1[33.6 ; 91.7]$ & 170 & $54.0[29.5 ; 86.5]$ & 99 & $69.4[36.6 ; 101.7]$ & 0.025 \\
\hline SAT index & 254 & $55.4[35.8 ; 75.5]$ & 155 & $51.5[35.1 ; 74.7]$ & 99 & $58.2[36.8 ; 77.9]$ & 0.1 \\
\hline Muscle HU & 403 & $34.6[27.5 ; 39.9]$ & 187 & $36.3[27.8 ; 42.4]$ & 216 & $32.6[26.6 ; 38.3]$ & $<0.001$ \\
\hline IMAT HU & 387 & $-58[-62 ;-55]$ & 171 & $-59[-62 ;-56]$ & 216 & $-58[-62 ;-54]$ & 0.039 \\
\hline VAT HU & 386 & $-88[-95 ;-80]$ & 170 & $-87[-93 ;-79]$ & 216 & $-90[-97 ;-82]$ & 0.003 \\
\hline SAT HU & 371 & $-98[-104 ;-89]$ & 155 & $-97[-103 ;-89]$ & 216 & $-98[-104 ;-90]$ & 0.48 \\
\hline \multicolumn{8}{|c|}{ Outcome } \\
\hline Death & 403 & $288(71.5 \%)$ & 187 & $112(59.9 \%)$ & 216 & $176(81.5 \%)$ & \\
\hline
\end{tabular}

BCLC, Barcelona Clinic Liver Cancer; BMI, Body mass index; HBV, chronic viral hepatitis B infection; HCV, chronic viral hepatitis $\mathrm{C}$ infection; HU, Hounsfield unit; IMAT, intermuscular adipose tissue; MELD, model for end-stage liver disease; NAFLD, non-alcoholic fatty liver disease; NK, unknown etiology; OE, other etiologies (haemochromatosis, primary biliary cholangitis, primary sclerosing cholangitis); SAT, subcutaneous adipose tissue; VAT, visceral adipose tissue

Index: $\mathrm{cm}^{2} / \mathrm{m}^{2}$

a) $\mathrm{N}$ represents the number of patients with available data; b) Continuous data are shown as median \pm interquartile range, categorical data as count and percentage

This article is protected by copyright. All rights reserved 
Table 2. Cox proportional hazard crude and adjusted model for survival in both cohorts

\begin{tabular}{|c|c|c|c|c|c|c|}
\hline 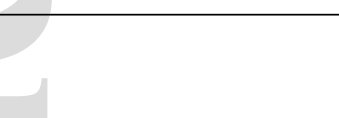 & \multicolumn{3}{|c|}{ Crude model } & \multicolumn{3}{|c|}{$\begin{array}{l}\text { Adjusted model for baseline } \\
\text { characteristics }\end{array}$} \\
\hline & $\mathrm{HR}$ & $\mathrm{Cl}$ & P-value & $\mathrm{HR}$ & $\mathrm{Cl}$ & P-value \\
\hline \multicolumn{7}{|l|}{ Bern cohort } \\
\hline BMI & 0.98 & {$[0.95,1.02]$} & 0.4 & 0.98 & {$[0.94,1.03]$} & 0.4 \\
\hline Muscle index ${ }^{\dagger}$ & 1.06 & {$[0.84,1.33]$} & 0.64 & 1.15 & {$[0.86,1.52]$} & 0.34 \\
\hline VAT index ${ }^{\dagger}$ & 0.97 & {$[0.92,1.02]$} & 0.27 & 0.96 & {$[0.9,1.02]$} & 0.16 \\
\hline SAT index ${ }^{\dagger}$ & 0.97 & {$[0.91,1.04]$} & 0.39 & 0.99 & {$[0.92,1.07]$} & 0.84 \\
\hline IMAT index $\log ^{\dagger}$ & 1.11 & {$[0.88,1.41]$} & 0.36 & 1.05 & {$[0.8,1.39]$} & 0.71 \\
\hline Muscle density $(\mathrm{HU})^{\dagger}$ & 0.92 & {$[0.77,1.1]$} & 0.38 & 1.05 & {$[0.85,1.29]$} & 0.66 \\
\hline${\text { IMAT density }(\mathrm{HU})^{\dagger}}^{\dagger}$ & 1.07 & {$[0.7,1.62]$} & 0.77 & 0.95 & {$[0.61,1.47]$} & 0.8 \\
\hline $\mathrm{VAT}$ density $(\mathrm{HU})^{\dagger}$ & 1.38 & {$[1.1,1.72]$} & 0.005 & 1.31 & {$[1.05,1.65]$} & 0.019 \\
\hline SAT density $(\mathrm{HU})^{\dagger}$ & 1.35 & {$[1.12,1.62]$} & 0.001 & 1.27 & {$[1.04,1.57]$} & 0.022 \\
\hline \multicolumn{7}{|l|}{ Newcastle cohort } \\
\hline BMI & 0.98 & {$[0.95,1.01]$} & 0.25 & 0.95 & {$[0.9,1.01]$} & 0.12 \\
\hline Muscle index ${ }^{\dagger}$ & 0.79 & {$[0.62,0.99]$} & 0.045 & 0.83 & {$[0.51,1.33]$} & 0.43 \\
\hline IMAT index $\log ^{\dagger}$ & 1.02 & {$[0.78,1.32]$} & 0.9 & 1.16 & {$[0.69,1.94]$} & 0.58 \\
\hline VAT index ${ }^{\dagger}$ & 0.95 & {$[0.9,1]$} & 0.051 & 1.03 & {$[0.95,1.11]$} & 0.53 \\
\hline SAT index ${ }^{\dagger}$ & 0.96 & {$[0.9,1.02]$} & 0.17 & 1.01 & {$[0.89,1.14]$} & 0.92 \\
\hline Muscle $\mathrm{HU}^{\dagger}$ & 0.87 & {$[0.73,1.04]$} & 0.14 & 0.79 & {$[0.59,1.06]$} & 0.12 \\
\hline IMAT_HU $^{\dagger}$ & 1.21 & {$[0.91,1.6]$} & 0.19 & 0.62 & {$[0.39,0.96]$} & 0.034 \\
\hline $\mathrm{VAT}$ density $(\mathrm{HU})^{\dagger}$ & 1.53 & {$[1.3,1.81]$} & $<0.001$ & 1.27 & {$[0.99,1.62]$} & 0.061 \\
\hline
\end{tabular}

This article is protected by copyright. All rights reserved 
BMI, body mass index; $\mathrm{Cl}$, confidence interval; HR, hazard ratio; HU, Hounsfield unit; IMAT, intermuscular adipose tissue; SAT, subcutaneous adipose tissue; VAT, visceral adipose tissue;

Index: $\mathrm{cm}^{2} / \mathrm{m}^{2}$

† Variable expressed per 10 units 


\section{Legend for Figure}

Figure 1. Kaplan-Meier survival curves in patients with hepatocellular carcinoma stratified according to subcutaneous adiposity (below and above median).

Kaplan-Meier survival curves estimating the overall survival (OS) in patients with hepatocellular carcinoma (HCC) stratified according to subcutaneous adipose tissue (SAT) density (high vs. low) at the level of the third lumbar vertebra (L3) as determined by median -97 Hounsfield units (HU) for Bern cohort and -98 HU for Newcastle cohort. Log rank test in Bern, $p=0.042$; in Newcastle, $p=0.003$. 
(A) Bern

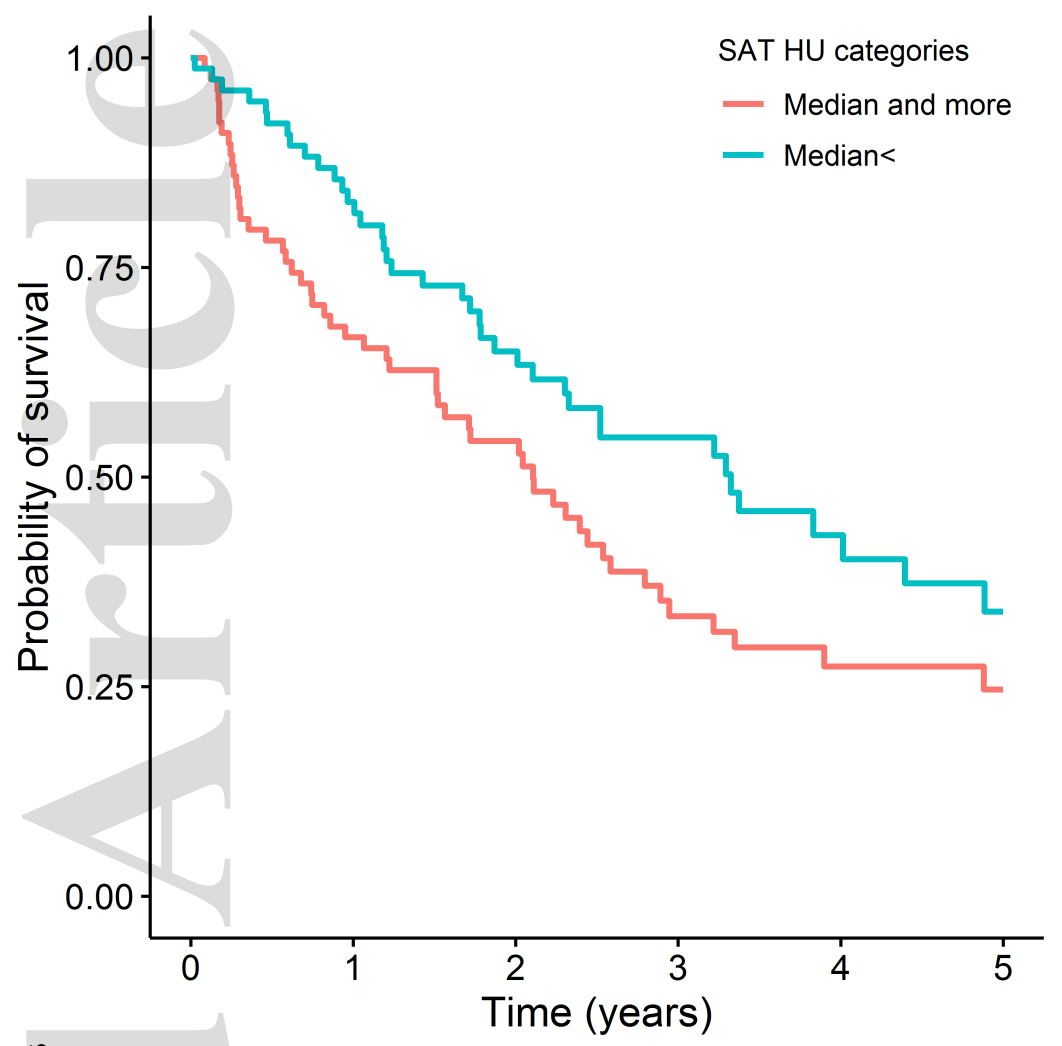

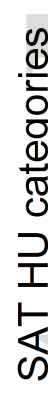

(B) Newcastle

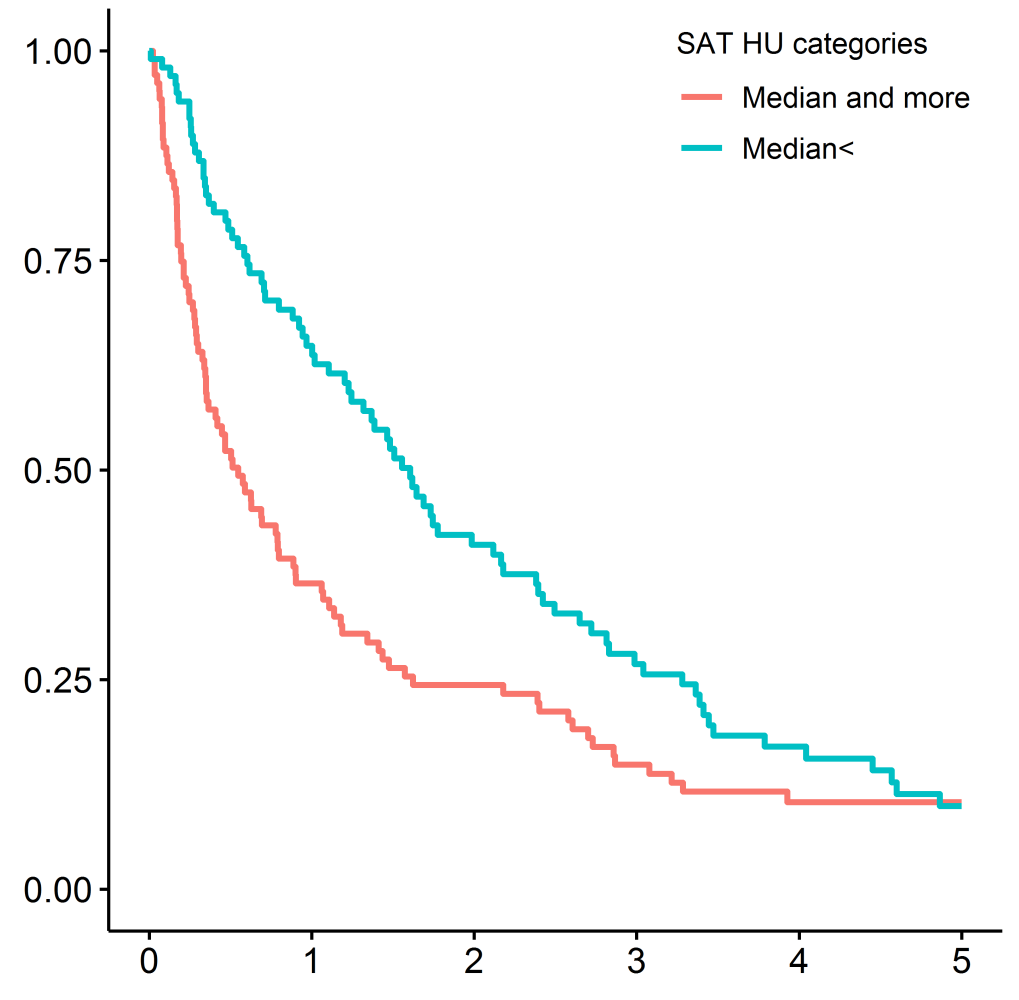

liv_14755_f1.eps 